\title{
Relay-Aided Outdoor-to-Indoor Communication in Millimeter-Wave Cellular Networks
}

\author{
Konstantinos Ntontin, Member, IEEE and Christos Verikoukis, Senior Member, IEEE
}

\begin{abstract}
In this work, we examine the feasibility of serving an indoor mobile terminal (MT) by an outdoor base station (BS) in urban millimeter-wave (mmWave) cellular networks. Towards this, we consider two scenarios: i) The signal reaches the reference MT directly by penetrating the building where it is located; ii) The communication is assisted by a relay placed on the rooftop of the building that conveys through a waveguide the desired signal to an indoor transmit antenna located at the floor where the reference MT is located. By using a stochastic-geometry based framework, we derive for both scenarios analytical expressions for the coverage and average rate, which exhibit a good match with respect to the simulations. In addition, from these expressions we gain important insights regarding the performance comparison of the two scenarios. In particular, it is analytically proved and validated by the simulations that relaying notably enhances the performance of the low-rate MTs even under substantial waveguide losses. However, for such losses the average rate in the relaying case can become worse than the case without relays.
\end{abstract}

Index Terms-Millimeter-Wave Cellular Networks, Outdoorto-Indoor Communication, Relays, Poisson Point Processes, Coverage Rate, Average Rate.

\section{INTRODUCTION}

Over the last years, there has been a continuous increase in the demand for higher rates, which has been pushed forward by the proliferation of smartphone devices. According to the overwhelming figures, it is expected that by 2021 global mobile data traffic will reach a monthly run of around 49 exabytes per year, which corresponds to a 7-fold growth with respect to the monthly run of 2016 [1]. Obviously, such demands cannot be accommodated by the current cellular standards that rely on the sub- $6 \mathrm{GHz}$ bands to convey information, such as the Long-Term Evolution Advanced, which can offer a peakdata rate slightly above $1 \mathrm{Gbps}$ [2]. Hence, the future of the substantially congested sub-6 GHz bands that are used for communication is highly uncertain. Due to this, an integral feature of $5 \mathrm{G}$ approaches that have been proposed to meet the ever-increasing rate demands has been the migration to higher frequencies due to the availability of a significantly higher bandwidth [3]. Such an availability directly translates to higher rates according to Shannon's formula.

Manuscript received February 03, 2019; revised July 172019 ; accepted August 25, 2019. This work was supported in part by AGAUR (2017-SGR 891) together with the 5G STEP-FWD (722429) and SPOT5G (TEC201787456-P) projects.

K. Ntontin is with the Institute of Informatics and Telecommunications, National Center for Scientific Research, "Demokritos", Greece (e-mail: konstantinos.ntontin@iit.demokritos.gr).

C. Verikoukis is with the SMARTECH Department, Telecommunications Technological Centre of Catalonia (CTTC/CERCA), Spain (e-mail: cveri@cttc.es).
Regarding the use of above- $6 \mathrm{GHz}$ bands for communication, there has been substantial research over the recent years regarding the potential of cellular communications at millimeter-wave (mmWave) bands that span the range between 30 and $100 \mathrm{GHz}$. Such frequencies are subject to a higher path loss compared to the currently-used sub- $6 \mathrm{GHz}$ bands. However, their smaller wavelength enables the placement of a higher number of antennas to the same physical space compared to their sub- $6 \mathrm{GHz}$ counterparts. As a result, a higher directionality through beamforming can be achieved between a mobile terminal (MT) and its associated base station (BS), which can counteract the larger free-space path loss [4]. As far as works which show such a potential are concerned, outdoor and indoor channel models have been developed that apply to particular carrier frequencies, such as the 28 and 73 $\mathrm{GHz}$ bands [4], [5]. According to these works, both coverageprobability and average-rate gains can be achieved compared to sub- $6 \mathrm{GHz}$ networks, as the density of the BSs increases and for highly-directional antennas employed at both the BSs and MTs. In addition, outdoor measurements in urban scenarios show that distances between a MT and its associated BS smaller than $200 \mathrm{~m}$ are needed so that outage-free communication is achieved [4]. Hence, the migration to mmWave bands is essential to be combined with cell densification in order to leverage the substantially higher available bandwidth [6]. Otherwise, relaying can be used to assist the BS-MT communication if high cell densification is not feasible [79].

The aforementioned works, which validate the mmWavecommunication potentials for achieving the rates foreseen by the $5 \mathrm{G}$ technology, examine only point-to-point systems. However, for the system designer it would be also important to be in possession of a tractable framework regarding the performance that can be achieved by considering the whole network topology and the possible sources of interference. Towards this, in [10] and [11] the authors provide analytical expressions for the coverage probability, coverage rate, and average rate by considering a stochastic-geometry approach where the BSs and centers of blockages are modeled on the plane as points of a Poisson point process (PPP) [12]. In addition, other indicative works on the area are [13-16].

As far as outdoor-to-indoor communication at mmWave bands is concerned, measurements have shown that mmWave signals are subject to substantial penetration losses as they penetrate common building materials. More specifically, penetration losses of around 40 and $28 \mathrm{~dB}$ have been reported for buildings made of tinted-glass and brick, respectively [17]. Such high penetration losses for common building materials are also verified in other works [18, and referenes therein]. 
Due to this, in [19] the authors suggest that it would be unlikely to have indoor MTs being served by outdoor mmWave BSs. Instead, coverage only by indoor mmWave BSs would seem the only viable option. However, such an option poses a significant challenge for the backhaul network accommodating the indoor BSs due to the high data rates stemming from the large available bandwidth [14]. Deploying fiber-optic cables reaching every building and every indoor BS at the different floors could potentially solve this issue, but the cost may be prohibitive, especially for dense urban areas [20], [21]. Consequently, wireless solutions seem more promising [22].

Contribution: Against the presented background, in this work we study the feasibility of allowing indoor MTs to be served by outdoor BSs at mmWave bands and provide the following threefold contribution:

- Firstly, we consider the direct BS-MT communication through building penetration. By incorporating the signal dissipation caused by the penetration into the path-loss model and by using a stochastic-geometry approach for the modeling of the location and number of BSs and buildings, we derive a one-fold integral expression for the achieved coverage rate and a two-fold integral expression for the average rate.

- Secondly, owing to the substantial penetration loss by common building materials, we propose the deployment of decode-and-forward (DF) [23] rooftop relays (one per building) that assist the BS-MT communication. In particular, the signal conveyed by the associated BS to the rooftop relay of the reference building is decoded, remodulated, and waveguided to one of its transmit antennas located at the reference floor, which wirelessly dispatches it to the reference MT. By incorporating as a parameter of the path-loss model the losses caused by the waveguide, we derive a closed-form expression for the achieved coverage rate and a one-fold integral expression for the average rate.

- Finally, based on the derived analytical expressions, we extract important insights regarding the coverage-rate comparison between the communication without relays and the one that involves relaying. In particular, we prove that relaying offers a notably higher coverage rate for the low-rate MTs, even under substantial waveguide losses and the worst-case scenario for the propagation conditions of the BS-relay links.

The aforementioned trends are validated by means of Monte-Carlo simulations. In addition, the simulations show that a substantial performance gain is achieved by relaying when the waveguide losses are small to moderate. On the other hand, for substantial waveguide losses the average rate of the relaying case can become smaller than the corresponding one of the case without relays.

Organization: The rest of this paper is organized as follows: In Section II, we present the network-layout assumptions together with the assumptions for each of the two examined cases. In Section III, we present the instantaneous SINR expressions and mathematically formulate the expressions for the coverage and average rate for both cases. In Section IV, we derive the analytical expressions of the examined metrics and, based on these, we provide insights regarding their coveragerate comparison. In Section $\mathrm{V}$, the analytical insights are validated by means of Monte Carlo simulations and, finally, Section VI concludes this work.

\section{SySTEM MODEL}

In this section, we present the main assumptions of the network layout together with the assumptions that correspond to each of the two considered cases, namely without and with relays.

\section{A. Network Layout}

The assumptions regarding the considered network model are the following:

Examined Indoor MT: We assume that the MT of interest is located at the center of a two-dimensional circular building with center the origin of $\mathbb{R}^{2}$. In addition, for the typical building we assume that its radius is denoted by $R_{B}$.

Blockage Modeling: Similar to [24], for the blockages that may exist in the radio path we consider that they are described by a Boolean scheme of circles on $\mathbb{R}^{2}{ }^{1}$. According to this assumption, the centers of the blockages form a homogeneous PPP $\Phi_{\text {Blockages }}$ of intensity $\lambda_{\text {Blockages. }}$ In addition, for simplicity we assume that the radius of all the buildings is equal to $R_{B}^{2}$.

By denoting by $K_{i}$ the number of blockages in a random link $i$ from a BS to the circumference of the reference building and by $R_{i}$ the length of the link, it is proved in [25], [26] that $K_{i}$ is a Poisson random variable with expected value equal to $\beta R_{i}$. Regarding $\beta$, it holds that $\beta=\frac{-\rho \ln (1-p)}{\pi A}$, where $\rho=2 \pi R_{B}$ and $A=\pi R_{B}^{2}$ are the perimeter and area of the buildings, respectively, and $p=\lambda_{\text {Blockages }} \pi R_{B}^{2}$, with $0<$ $p<1$, is the percentage of the area occupied by buildings. In addition, the probability that the link $i$ does not contain any blockage, which we denote by $\operatorname{Pr}\left\{K_{i}\right\}$, is given by

$$
\operatorname{Pr}\left\{K_{i}=0\right\}=\exp \left(-\beta R_{i}\right) .
$$

Finally, for analytical tractability we assume that the number of blockages in any two links from two BSs to the MT of interest are independent. This means that the same blockage cannot be in the radio path of more than one link from a BS to the reference MT. According to [25], such a simplification causes a minor loss in accuracy when the size of the blockages is relatively small.

BS Modeling: We only consider outdoor BSs for which we assume that they are uniformly distributed on $\mathbb{R}^{2}$ according to a PPP $\Phi_{B S}$ with intensity $\lambda_{B S}=1 /\left(\pi R_{\text {cell }}^{2}\right)$, where $R_{\text {cell }}$ is the average cell radius. Furthermore, we assume that $\Phi_{B S}$ and $\Phi_{\text {Blockages }}$ are independent.

Frequency Band of Operation: We consider the $28 \mathrm{GHz}$ mmWave band for operation. Such a band has been considered in several literature work as a candidate for future mmWave access networks [4], [6].

\footnotetext{
${ }^{1}$ As it is pointed out in [24], the reasons for choosing the circular-building modeling are its smaller mathematical complexity compared to the randomshape building modeling and the fact that the insights obtained by such a more realistic consideration are not expected to differ.

${ }^{2}$ Such an assumption has been also considered in [24].
} 


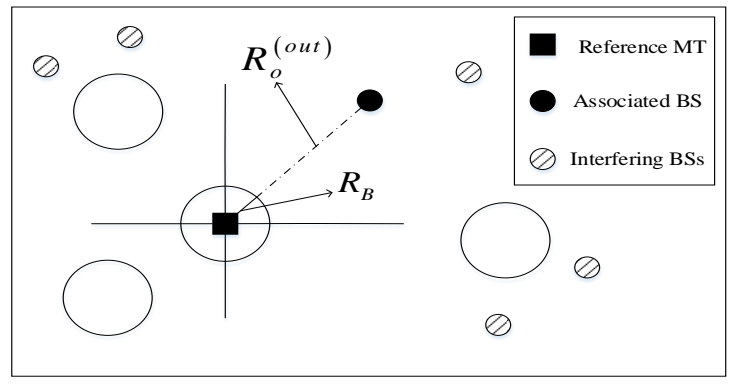

Fig. 1: Depiction of $R_{o}^{(o u t)}$. The large circles represent the buildings.

Propagation Conditions: We assume that the outdoor mmWave communication can occur in line-of-sight (LOS) and non-LOS (NLOS) conditions, which is supported by reported measurements [5], [11]. In addition, measurements also justify the consideration of an outage state, denoted by $P_{\text {threshold }}$, which occurs when the received signal power is smaller than a minimum threshold that is needed for both detection and data acquisition [4], [27]. The occurrence of the outage state will be analytically described later in this section.

Access Protocol: We assume that the outdoor and indoor users are served at different allocated time periods in a roundrobin fashion. Furthermore, we assume that the downlink access within a building occurs in a time-division multiple access (TDMA) fashion. This means that at each time slot only one MT per reference building is scheduled ${ }^{3}$ for transmission, for which the whole available bandwidth is given. In addition, if more than one buildings are associated with the same BS, the different buildings and, consequently, their MTs are also served in a TDMA fashion ${ }^{4}$.

Outdoor Path-Loss Considerations: We denote the distance between the associated BS and the radial point located at the circumference of the reference building by $R_{o}^{(o u t)}$. This is depicted in Fig. 1. Moreover, the 'close-in' path-loss model is considered in our work [6]. Finally, we assume that the attenuation of the electromagnetic signal as it propagates due to the gases in the atmosphere is negligible compared to the path loss. Such an assumption would be valid for typical distances between the center of the reference building and its associated BS that are likely to be encountered in a small-cell scenario and for frequencies below $100 \mathrm{GHz}$ that we consider in this work. This is evident according to [6, Fig. 2], which illustrates the atmospheric attenuation versus frequency.

Antenna gains of the BSs and MTs: We assume that antenna arrays are deployed at the BSs and MTs in order to enable beamsteering. Their gains are given by a sectored radiation pattern as $[10]^{5}$ :

$$
G_{q}(\theta)= \begin{cases}G_{q}^{(\max )}, & |\theta| \leq \theta_{q} \\ G_{q}^{(\min )}, & |\theta|>\theta_{q}\end{cases}
$$

\footnotetext{
${ }^{3}$ Studying different scheduling criteria is out of the scope of this work.

${ }^{4}$ We require that only one MT per building is scheduled at each time slot for the following two reasons: i) In order to avoid possible interference among adjacent floors; ii) In order to avoid the need for the transmit electronics of the BS to be equipped with more than one radio-frequency (RF) chains so to concurrently generate different data streams for different MTs.

${ }^{5}$ Antenna arrays with important gains that are suitable for the size of MTs and are based on both dipoles and patch antennas have been proposed in the literature [28], [29].
}

$q \in\{B S, M T\}, \theta \in[-\pi, \pi)$ is the angle off the antenna boresight direction, $\theta_{q}$ is the half-power beamwidth of the main lobe, and $G_{q}^{(\max )}, G_{q}^{(\min )}$ are the maximum and minimum antenna gains, respectively. Hence, as it is also assumed in [10], with such modeling for simplicity we assume that the array gains are constant and equal to $G_{q}^{(\max )}$ and $G_{q}^{(\min )}$ for all angles in the main lobe and side lobes, respectively.

Shadowing: For analytical tractability, we assume that the possible shadowing effects are incorporated into the blockage model, as it is assumed in [13].

Load Scenario: We consider a saturated-load scenario where the number of frequency bands that each BS has at its disposal is smaller than the number of MTs it has to serve. Hence, we assume that all the BSs other than the serving BS are interferers towards the reference MT.

\section{B. Case Without Relays}

Cell Association: For simplification and considering that our aim in this work is to examine trends that are expected not to depend on the considered cell-association type, we assume that each MT is served by its closest outdoor BS that is located outside the area of the reference building.

Building Penetration Loss: We assume that the material penetration loss of the reference building is denoted by $\gamma$. Indicative values of $\gamma$ for different materials, such as tinted glass and brick, for the mmWave band of $28 \mathrm{GHz}$ are given in [17].

Outdoor-to-Indoor Path Loss: To the best of our knowledge, only [30] and [31] consider a mathematical model for such scenarios, which consists of the addition to an outdoor path loss model of the losses related to the material penetration loss and the internal wall losses. This is the model that we also consider in our work ${ }^{6}$. In particular, let us assume that the link between a BS and the reference MT is denoted by $i$ and the distance between them is equal to $R_{i}^{(o u t)}+R_{B}$. The resulting large-scale path loss in the cases of outdoor LOS and NLOS links, which we denote by $l_{s_{1}}\left(R_{i}^{(\text {out })}, R_{B}\right)$, where $s_{1} \in\{L O S, N L O S\}$, is given by

$$
l_{s_{1}}\left(R_{i}^{(\text {out })}, R_{B}\right)=\kappa_{0} \gamma\left(R_{i}^{(\text {out })}+R_{B}\right)^{\alpha_{s_{1}}} L_{i n},
$$

where $\alpha_{s_{1}}$, denotes the path-loss exponent in the case of outdoor LOS and NLOS links, fitted to the outdoor-to-indoor propagation measurements, and $L_{i n}$ are the losses due to wall penetrations inside the floor where the reference MT is located ${ }^{7}$. In addition, $\kappa_{0}$ denotes the free-space path loss at a distance of $1 \mathrm{~m}$. It holds that $\kappa_{0}=(4 \pi f / c)^{2}$, where $f$ is the carrier frequency. Hence, in linear scale it holds that

$$
l_{s_{1}}\left(R_{i}^{(\text {out })}, R_{B}\right)=\kappa_{0} \gamma\left(R_{i}^{(\text {out })}+R_{B}\right)^{\alpha_{s_{1}}} L_{i n} .
$$

Beamsteering: We assume that prior to data transmission the MT and its serving BS steer the orientations of their

\footnotetext{
${ }^{6}$ Including additional factors to an outdoor propagation model that correspond to the material penetration internal wall losses inside the building might not be the best approach for modeling such channels, as it is pointed out in [18], but, to our understanding, it is a valid rough approach for modeling that can give some important trends regarding different material types.

${ }^{7}$ Considering the parameter $L_{i n}$ that represents the losses of the electromagnetic wave as it penetrates indoor walls is consistent with measurements at the $28-\mathrm{GHz}$ band [17].
} 
TABLE I: Possible directivity gains of the interfering links and associated probabilities of occurrence.

\begin{tabular}{|l|l|l|}
\hline Direct. Gain & $G_{B S}^{(\max )} G_{M T}^{(\max )}$ & $G_{B S}^{(\max )} G_{M T}^{(\min )}$ \\
\hline Probability & $F_{B S} F_{M T}$ & $F_{B S}\left(1-F_{M T}\right)$ \\
\hline \multicolumn{3}{|c|}{} \\
\hline Direct. Gain & $G_{B S}^{(\min )} G_{M T}^{(\max )}$ & $G_{B S}^{(\min )} G_{M T}^{(\min )}$ \\
\hline Probability & $\left(1-F_{B S}\right) F_{M T}$ & $\left(1-F_{B S}\right)\left(1-F_{M T}\right)$ \\
\hline
\end{tabular}

antennas in a way that the maximum directivity gain, i.e. $G_{B S}^{(\max )} G_{M T}^{(\max )}$, is achieved. This can be realized by the existence of a feedback channel and the dispatch of pilot signals from the MT to its serving BS prior to data transmission. Since the beams of the interfering links are uniformly distributed in $[-\pi, \pi)$, there are 4 possible values of the directivity gain between an interferer and the MT of interest, i.e. $G_{B S}^{(\max )} G_{M T}^{(\max )}, G_{B S}^{(\max )} G_{M T}^{(\min )}, G_{B S}^{(\min )} G_{M T}^{(\max )}$, and $G_{B S}^{(m i n)} G_{M T}^{(m i n)}$ with respective probabilities given in Table I. $F_{B S}=\frac{\theta_{B S}}{2 \pi}$ and $F_{M T}=\frac{\theta_{M T}}{2 \pi}$ are the probabilities that an interfering BS and the MT of interest are aligned in a direction that provides antenna gains $G_{B S}^{(\max )}$ and $G_{M T}^{(\max )}$, respectively.

Outage Case: As we have aforementioned in Section II-A, if the received power is smaller than $P_{\text {threshold }}$, either the signal cannot be detected or can be detected, but cannot be demodulated [27]. In such a case, the SINR is equal to 0. Correspondingly, there is a cutoff distance over which the signal is in outage, which we denote by $R_{r e f}^{s_{1}}, s_{1} \in\{L O S, N L O S\}$, for each of the 2 outdoor propagation possibilities. These distances can be found from the link budget as

$$
\begin{aligned}
P_{\text {threshold }}[d B] & =10 \log _{10}\left(P_{T} G_{B S}^{(\max )} G_{M T}^{(\max )}\right) \\
& -10 \log _{10}\left[l_{s_{1}}\left(R_{r e f}^{s_{1}}, R_{B}\right)\right] \\
& \Rightarrow R_{\text {ref }}^{s_{1}}=\left(\frac{P_{T} G_{B S}^{(\max )} G_{M T}^{(\max )}}{P_{\text {threshold }} \kappa_{0} \gamma L_{\text {in }}}\right)^{\frac{1}{\alpha_{s_{1}}}}-R_{B} .
\end{aligned}
$$

Fast Fading: We assume that the small-scale movement of the reference MT inside the reference building is subject to fast fading, denoted by $h$, with amplitude following a Rayleigh distribution with unit variance of the corresponding complex envelope. Hence, it holds that $h \sim C N(0,1)$.

\section{Case With Relays}

Relay Deployment and Cell Association: Due to the large value of $\gamma$ for common building materials [17], we propose to deploy one DF relay per rooftop. Such a relay enables the detected and remodulated mmWave signal to be conveyed through a waveguide to a transmit antenna mounted on an interior wall at the floor where the reference MT is located. Then, the signal is wirelessly dispatched to the reference $\mathrm{MT}^{8}$. Such a proposed architecture is illustrated in Fig. 2 for a typical building under indicative LOS and NLOS conditions regarding the link between the associated outdoor BS and the rooftop relay of the reference building. Consequently, the rooftop relays form a homogeneous PPP, denoted by $\Phi_{\text {Relays }}$, with intensity equal to $\lambda_{\text {Blockages }}$.

\footnotetext{
${ }^{8}$ The proposed architecture is similar to the distributed antenna system architecture for in-building wireless communications [32].
}

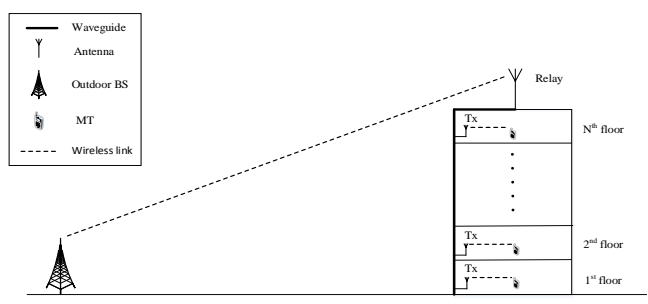

(a) LOS case.

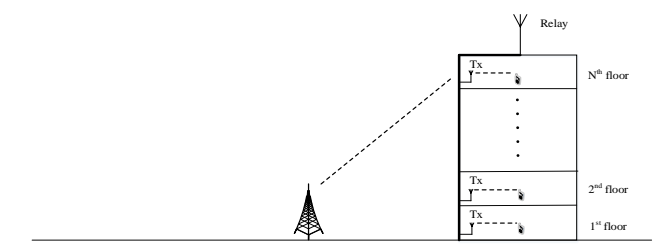

(b) NLOS case. Communication between the outdoor BS and the relay is feasible through reflections as measurements at mmWave bands show [4], [6].

Fig. 2: Proposed relaying architecture for the outdoor-to-indoor communication.

As far as the cell association is concerned, similar to the scenario without the relays we assume that the rooftop relay of the reference building is associated with its closest BS. In addition, the BS-relay communication is feasible if the link between the BS and the point in the circumference of the circular reference building (that is located at the radial distance between the BS and the reference MT) has a distance smaller than a cut-off distance, denoted by $R_{r e f}^{(R N)}$.

Waveguide Design: Based on the proposed relaying architecture, ideally we would require that losses associated with the propagation of the electromagnetic signal in the waveguide are much smaller compared to the outdoor path loss. Towards this, we are going to present two architectures that can make such a design feasible: radio over fiber and silicon-ribbon waveguide.

1) Radio Over Fiber: According to the radio-over-fiber approach, the waveguide is an optical fiber cable. Based on this, at the relay the modulated mmWave signal modulates an optical carrier via an electrical-to-optical conversion process [33]. Such a process can be performed in the analog or digital domain. In the latter case, the modulated mmWave signal is digitized before modulating the optical carrier [33]. At the end of the fiber cable, before the transmit antenna, the transmitted optical signal is detected and converted into an electrical mmWave signal through a photodetector. Considering that the propagation loss of an optical fiber is smaller than 5 $\mathrm{dB} / \mathrm{km}$ [34] and, consequently, negligible for typical building heights, the main losses are the ones that correspond to the efficiency of the electrical-to-optical and optical-to-electrical conversion processes. Such efficiency can reach $50 \%$ with advanced designs [35].

2) Silicon-Ribbon Waveguide: According to the siliconribbon waveguide approach, the waveguide operates directly at the mmWave band of interest and it is made of silicon ribbon. Such a waveguide exhibits a very small propagation loss, in 
the order of $0.014 \mathrm{~dB} / \mathrm{m}$ for the $28 \mathrm{GHz}$ band, according to theoretical studies [36, Fig. 1]. Hence, the propagation loss can be considered negligible for typical building heights compared to the outdoor path loss.

Remark 1: We note that the radio-over-fiber approach is an already deployed approach for dispatching radio signals through a high-bandwidth optical cable. Hence, the required equipment including the electronics and cables are readily available. In contrast, silicon-ribbon waveguides with a very small propagation loss, as the one presented in [36], have only been the subject of theoretical modeling. To the best of the authors' knowledge, such waveguides have not been manufactured yet. Based on these, we model the waveguide associated losses that include every possible loss, such as propagation losses, connector losses, and electrical-to-optical/optical-toelectrical conversion losses (in the case of an optical-fiber waveguide) as a parameter that we denote by $L_{\text {wav }}$.

Relay Operational Principle: We assume that the relays follow the half-duplex (HD) principle, which means that they cannot receive and transmit at the same time slot [23]. In particular, we assume that the communication is divided into the following two phases with duration one time slot each: i) In the 1 st phase, only the BSs are allowed to transmit, which dispatch their packets to the rooftop relays; ii) In the 2 nd phase, only the relays are allowed to operate, which dispatch their packets to the indoor MTs through the indoor transmit antennas. Consequently, the symbol rate is 0.5 symbols/time slot, which is half of the direct communication without relays between the BS and the reference MT.

Antenna Gain of the Relays and Beamsteering: As in the case of the BSs and MTs, we assume that antenna arrays are deployed for transmission and reception at the rooftop relays. Their gains follow the sector pattern of (2), with gains denoted by $G_{R N_{(R x)}}^{(m)}$ and $G_{R N_{(T x)}}^{(m)}$, where $m \in[\max , \min ]$, for transmission and reception, respectively. In addition, we assume that the boresight directions for both the BS-relay and relay-MT transmissions are aligned and, consequently, the maximum directivity gain is achieved. Similar to the case without relays, there are 4 possible values of the directivity gain between an interferer and the relay, i.e. $G_{B S}^{(\max )} G_{R N_{(R x)}}^{(\max )}$, $G_{B S}^{(\max )} G_{R N_{(R x)}}^{(\min )}, G_{B S}^{(\min )} G_{R N_{(R x)}}^{(\max )}$, and $G_{B S}^{(\min )} G_{R N_{(R x)}}^{(\min )}$ with respective probabilities $F_{B S} F_{R N_{(R x)}}, F_{B S}\left(1-F_{R N_{(R x)}}\right)$, $\left(1-F_{B S}\right) F_{R N_{(R x)}}$, and $\left(1-F_{B S}\right)\left(1-F_{R N_{(R x)}}\right)$, where $F_{R N_{(R x)}}=\frac{\theta_{R N}(R x)}{2 \pi}$ with $\theta_{R N_{(R x)}}$ being the half-power beamwidth of the main lobe.

Similarly, regarding the transmission from the transmit antenna of the relay at the second time slot, there are 4 possible values of the directivity gain between an interfering relay and the indoor reference MT, i.e. $G_{R N_{(T x)}}^{(\max )} G_{M T}^{(\max )}$, $G_{R N_{(T x)}}^{(\max )} G_{M T}^{(\min )}, \quad G_{R N_{(T x)}}^{(\min )} G_{M T}^{(\max )}, \quad$ and $G_{R N_{(T x)}}^{(\min )} G_{M T}^{(\min )}$ with probabilities $F_{R N_{(T x)}} F_{M T}, \quad F_{R N_{(T x)}}\left(1-F_{M T}\right)$, $\left(1-F_{R N_{(T x)}}\right) F_{M T}, \quad$ and $\quad\left(1-F_{R N_{(T x)}}\right)\left(1-F_{M T}\right)$, respectively, where $F_{R N_{(T x)}}=\frac{\theta_{R N_{(T x)}}}{2 \pi}$ with $\theta_{R N_{(T x)}}$ being the half-power beamwidth of the main lobe.

Path Loss:

1) Outdoor Path Loss: The path loss between the rooftop relay and its closest $\mathrm{BS}$, which is denoted by $l_{s_{1}}^{(R N)}\left(R_{i}^{(\text {out })}, R_{B}\right)$, where $s_{1} \in\{L O S, N L O S\}$, is given by

$$
l_{s_{1}}^{(R N)}\left(R_{i}^{(\text {out })}, R_{B}\right)=\kappa_{0}\left(R_{i}^{(\text {out })}+R_{B}\right)^{\alpha_{s_{1}}^{(R N)}}
$$

where $\alpha_{s_{1}}^{(R N)}$ denotes the BS-relay path-loss exponent in the case of outdoor LOS and NLOS links.

2) Indoor Path Loss: We assume that the path loss, incorporating also the waveguide losses, between the indoor transmit antenna and the indoor reference MT located at the origin is given by

$$
l_{\text {in }}\left(R_{B}\right)=\kappa_{0} L_{w a v} F P L\left(R_{B}\right) L_{i n},
$$

where $F P L\left(R_{B}\right)$ is the indoor free-space path loss given by

$$
F P L\left(R_{B}\right)=10 \log _{10}\left(R_{B}^{2}\right) .
$$

Such a modeling for the indoor path loss is consistent with literature indoor measurements in which the path loss is divided into the free-space path loss and the loss associated with indoor obstacles [17, Table III].

Channel Quality of the BS-Relay Links: Due to the fact that we consider relays deployed on the rooftops of buildings, it may occur that the link between the relay of the typical building and its associated BS is a NLOS link even if there are no blockages between the BS and the reference building. This can be the case if the relay is mounted on a short mast. On the other hand, for tall masts it is expected that there is always LOS visibility between them if there are no blockages intercepting the line that connects them. Due to this, we consider the following two bounds in the modeling:

i) The Best-Case scenario: In this scenario, the BS-relay link is always a LOS link if there is no blockage intercepting the line that connects the BS with the relay.

ii) The Worst-Case scenario: In this scenario, the BS-relay link is always a NLOS link regardless of whether there is a blockage or not intercepting the line that connects the BS with the relay.

It is expected that in real-world scenarios the achieved performance would lie in between the corresponding performance of these two bound cases since some of the BS-relay links would be LOS and other NLOS.

Outage Case: Due to the fact that the communication link between the relay located on the rooftop of the reference building and its associated BS can be either LOS or NLOS, $R_{r e f}^{(R N)}$ takes two values, which we denote by $R_{r e f}^{s_{1}(R N)}$. These can be found by the link budget as

$$
\begin{aligned}
P_{\text {threshold }}[d B] & =10 \log _{10}\left(K_{0} P_{T} G_{B S}^{(\max )} G_{R N_{(R x)}}^{(\max )}\right) \\
& -10 \log _{10}\left[l_{s_{1}}^{(R N)}\left(R_{r e f}^{s_{1}(R N)}, R_{B}\right)\right] \\
& \Rightarrow R_{\text {ref }}^{s_{1}(R N)}=\left(\frac{K_{0} P_{T} G_{B S}^{(\max )} G_{R N_{(R x)}}^{(\max )}}{P_{\text {threshold }} \kappa_{0}}\right)^{\frac{1}{\alpha_{s_{1}}^{(R N)}}} \\
& -R_{B},
\end{aligned}
$$

where $K_{0}, 0<K_{0}<1$, is a scaling factor of the BSs transmit power. In addition, it holds that the transmit power of the relays is $\left(1-K_{0}\right) P_{T}$ so that the total transmit power 
of a BS-relay pair is $P_{T}$

Fast Fading: Due to the lack of mobility regarding the link corresponding to the communication between the outdoor serving BS and the rooftop relay of the reference building, we assume that there is no fast fading affecting the particular link. On the other hand, due to the small-scale mobility involved in the link that corresponds to the indoor communication between the rooftop relay and the reference MT, we assume that the particular indoor link is subject to fast fading, denoted by $h^{(R N)}$, with amplitude following a Rayleigh distribution with unit variance of the corresponding complex envelope. Hence, it holds that $h^{(R N)} \sim C N(0,1)$.

\section{InStantaneous SINR AND EXAMINED Metrics}

In this section, we first present the corresponding expressions of the instantaneous SINRs. Subsequently, we formulate the coverage and average rate as the metrics of interest.

\section{A. Instantaneous SINR}

1) Case Without Relays: In the case without relays, the received SINR at the reference MT, which we denote by $S I N R_{s_{1}}$, where $s_{1} \in\{L O S, N L O S\}$, is given by

$$
S I N R_{s_{1}}=\frac{P_{T}|h|^{2} G_{B S}^{(\max )} G_{M T}^{(\max )} / l_{s_{1_{o}}}\left(R_{o}^{(\text {out })}, R_{B}\right)}{I_{1}+I_{2}+I_{3}+I_{4}+N_{0}} .
$$

$N_{0}=-174+10 \log _{10}(B W)+N F(d B m)$ is the thermal noise with $B W$ and $N F$ being the used bandwidth and noise figure, respectively, and

$$
I_{m}=\sum_{\ell_{m}, \ell_{m} \neq o} P_{T}\left|h_{\ell_{m}}\right|^{2} G_{B S}^{(\max )} G_{M T}^{(\max )} / l_{s_{m}^{\prime}}\left(R_{\ell_{m}}^{(\text {out })}, R_{B}\right)
$$

with $R_{\ell_{m}}^{(\text {out })}>R_{o}^{(\text {out })} \forall \ell_{m} \neq o$ and $s_{m}^{\prime} \in\{L O S, N L O S\}$. $\left|h_{\ell_{m}}\right|$ are the fast-fading gains of the interfering links.

2) Case With Relays: Let us now present the instantaneous SINR expressions of the two phases in the case of relaying:

a) 1st Phase-Transmission from the outdoor associated $B S$ to the rooftop relay: During this phase, where only the BSs are allowed to transmit, the rooftop relay is subject to interference due to the transmissions of the rest of the outdoor BSs. Hence, the resulting SINR, which we denote by $S I N R_{s_{1}}^{(1)(R N)}$, is given by

$$
S I N R_{s_{1 o}}^{(1)(R N)}=\frac{K_{0} P_{T} G_{B S}^{(\max )} G_{R N_{(R x)}}^{(\max )} / l_{s_{1}}^{(R N)}\left(R_{o}^{(o u t)}, R_{B}\right)}{I_{1}^{(B S)}+I_{2}^{(B S)}+I_{3}^{(B S)}+I_{4}^{(B S)}+N_{0}},
$$

where

$$
I_{m}^{(B S)}=\sum_{\ell_{m}, \ell_{m} \neq 0} K_{0} P_{T} G_{B S}^{(\max )} G_{R N_{(R x)}}^{(\max )} / l_{s_{m}^{\prime}}^{(R N)}\left(R_{\ell_{m}}^{(\text {out })}, R_{B}\right)
$$

with $R_{\ell_{m}}^{(\text {out })}>R_{o}^{(o u t)} \forall \ell_{m} \neq o$ and $s_{m}^{\prime} \in\{L O S, N L O S\}$, where $m=\{1,2,3,4\}$.

b) 2nd Phase-Transmission from the relay to the MT:

This transmission occurs indoors since the transmit antenna of the relay is located at the interior wall of the floor where the indoor reference MT is located. The possible interferers to the MT arise from the transmissions at the same frequency band of the rooftop-relay antennas located in other buildings. Considering the fact that in each case the signals from such transmissions are subject to at least two outdoor wall penetrations until they reach the reference MT, we can logically assume that their power effect is negligible. This assumption is validated in Section V. Therefore, the resulting SINR at the reference MT, which we denote by $S I N R_{s_{2}}^{(2)(R N)}$, is given by

$S I N R^{(2)(R N)} \simeq \frac{\left(1-K_{0}\right) P_{T}\left|h^{(R N)}\right|^{2} G_{R N_{(T x)}}^{(\max )} G_{M T}^{(\max )} / l_{i n}\left(R_{B}\right)}{N_{0}}$,

\section{B. Coverage Rate}

1) Case Without Relays: In the case without relays, the coverage rate, which we denote by $R_{\text {cov }}\left(R_{t}\right)$, is the probability that the instantaneous rate of the direct BS-MT links is higher than a threshold $R_{t}$. Hence, it holds that ${ }^{9}$

$$
\begin{aligned}
R_{\mathrm{cov}}\left(R_{t}\right) & =\sum_{s_{1_{o}}} \in\{L O S, N L O S\} \\
& P_{r}^{s_{1}} \operatorname{Pr}\left\{B W \log _{2}\left(1+S I N R_{s_{1_{o}}}\right)>R_{t}\right\} \\
& =\sum_{s_{1_{o}} \in\{L O S, N L O S\}} P_{r}^{s_{1}} \operatorname{Pr}\left\{S I N R_{s_{1_{o}}}>2^{\frac{R_{t}}{B W}}-1\right\}
\end{aligned}
$$

where $P_{r}^{L O S}$ and $P_{r}^{N L O S}$ are the LOS and NLOS probabilities, respectively, given by

$$
\begin{aligned}
P_{r}^{L O S} & =2 \pi \lambda_{B S} \exp \left(\beta R_{B}\right) \exp \left(\pi \lambda_{B S} R_{B}^{2}\right) \\
& \times \int_{R_{B}}^{R_{r e f}^{L O S}+R_{B}} x \exp (-\beta x) \exp \left(-\pi \lambda_{B S} x^{2}\right) d x \\
& =\exp \left(\beta R_{B}\right) \exp \left(\pi \lambda_{B S} R_{B}^{2}\right)\left(G_{L O S}^{(1)}+G_{L O S}^{(2)}\right) \\
P_{r}^{N L O S} & =2 \pi \lambda_{B S} \exp \left(\beta R_{B}\right) \exp \left(\pi \lambda_{B S} R_{B}^{2}\right) \\
& \times \int_{R_{B}}^{R_{r e f}^{N L O S}+R_{B}} x \exp (-\beta x) \exp \left(-\pi \lambda_{B S} x^{2}\right) d x \\
& =\exp \left(\beta R_{B}\right) \exp \left(\pi \lambda_{B S} R_{B}^{2}\right) \\
& \times\left(G_{N L O S}^{(3)}-G_{N L O S}^{(1)}-G_{N L O S}^{(2)}\right)
\end{aligned}
$$

with

$$
\begin{aligned}
G_{s_{1}}^{(1)} & =\exp \left[-R_{B}\left(\pi \lambda_{B S} R_{B}+\beta\right)\right] \\
& -\exp \left[-\left(R_{r e f}^{s_{1 o}}+R_{B}\right)\left(\pi \lambda_{B S}\left(R_{r e f}^{s_{1}}+R_{B}\right)+\beta\right)\right] \\
G_{s_{1}}^{(2)} & =\sqrt{\pi} \beta \exp \left(\frac{\beta^{2}}{4 \pi \lambda_{B S}}\right) \operatorname{erf}\left(\frac{2 R_{B} \pi \lambda_{B S}+\beta}{2 \sqrt{\pi \lambda_{B S}}}\right) \\
& -\sqrt{\pi} \beta \exp \left(\frac{\beta^{2}}{4 \pi \lambda_{B S}}\right) \operatorname{erf}\left(\frac{2\left(R_{r e f}^{s_{1}}+R_{B}\right) \pi \lambda_{B S}+\beta}{2 \sqrt{\pi \lambda_{B S}}}\right) \\
G_{N L O S}^{(3)} & =\frac{\exp \left(-\pi \lambda_{B S} R_{B}^{2}\right)-\exp \left(-\pi \lambda_{B S}\left(R_{B}+R_{r e f}^{N L O S}\right)^{2}\right)}{\exp \left(\beta R_{B}\right)},
\end{aligned}
$$

$s_{1_{o}} \in\{L O S, N L O S\}$.

2) Case With Relays: In the relaying case, coverage rate is the probability that both the instantaneous rates of the BS-relay and relay-MT links are higher than the threshold $R_{t}$. Denoting them by $R_{\text {cov }}^{(R N)(B C)}\left(R_{t}\right)$ and $R_{\text {cov }}^{(R N)(W C)}\left(R_{t}\right)$ for the Best and Worst-Case scenarios, respectively, they are given by (20), seen on the top of the next page. $P_{r}^{L O S(R N)}$ and $P_{r}^{N L O S(R N)}$ are the LOS and NLOS probabilities, respectively, in the case

${ }^{9} \mathrm{We}$ do not consider the division of the rate expression with the number of MTs, as it would be required in our considered TDMA protocol. We have considered such a simplification due to the fact that the same scaling of the rate with the number of MTs applies to both the cases without and with relays. 


$$
\begin{gathered}
R_{\mathrm{cov}}^{(R N)(B C)}\left(R_{t}\right)=\sum_{s_{1_{o}} \in\{L O S, N L O S\}} P_{r}^{s_{1_{o}}(R N)} \operatorname{Pr}\left\{\frac{B W}{2} \log _{2}\left(1+S I N R_{s_{1}}^{(1)(R N)}\right), \frac{B W}{2} \log _{2}\left(1+S I N R^{(2)(R N)}\right)>R_{t}\right\} \\
=\sum P_{r}^{s_{1 o}(R N)} \operatorname{Pr}\left\{S I N R_{s_{1}}^{(1)(R N)}, S I N R^{(2)(R N)}>2^{\frac{2 R_{t}}{B W}}-1\right\} \\
s_{1_{o} \in\{L O S, N L O S\}} \\
R_{\mathrm{cov}}^{(R N)(W C)}\left(R_{t}\right)=\operatorname{Pr}\left\{\frac{B W}{2} \log _{2}\left(1+S I N R_{N L O S}^{(1)(R N)}\right), \frac{B W}{2} \log _{2}\left(1+S I N R^{(2)(R N)}\right)>R_{t}\right\} \\
=\operatorname{Pr}\left\{S I N R_{N L O S}^{(1)(R N)}, S I N R^{(2)(R N)}>2^{\frac{2 R_{t}}{B W}}-1\right\},
\end{gathered}
$$

of relaying and are given by (18) by replacing $R_{\text {ref }}^{L O S}$ and $R_{r e f}^{N L O S}$ with $R_{r e f}^{L O S(R N)}$ and $R_{r e f}^{N L O S(R N)}$, respectively.

\section{Average Rate}

1) Case Without Relays: In the case without relays, the average rate, which we denote by $R_{a v g}$, is given by

$$
\begin{aligned}
& R_{a v g}=\sum_{s_{1_{o}} \in\{L O S, N L O S\}} P_{r}^{s_{1_{o}}} E_{S I N R_{1_{1}}}\left\{B W \log _{2}\left(1+S I N R_{s_{1_{o}}}\right)\right\} \\
& \quad \stackrel{(a)}{=} B W \log _{2}(e) \sum_{s_{1_{o}} \in\{L O S, N L O S\}} P_{r}^{s_{1}} \int_{0}^{\infty} \frac{\operatorname{Pr}\left\{S I N R_{s_{1_{o}}}>x\right\}}{1+x} d x
\end{aligned}
$$

where $E\{\cdot\}$ denotes average value and in $(a)$ we use [37, Eq. (38)].

2) Case With Relays: In the relaying case, the average rate, denoted by $R_{a v g}^{(R N)(B C)}$ and $R_{a v g}^{(R N)(W C)}$ in the Best- and Worst-Case scenarios, respectively, is given by

$$
\begin{aligned}
& R_{\text {avg }}^{(R N)(B C)}=\sum_{s_{1_{o}} \in\{L O S, N L O S\}} P_{r}^{s_{1_{o}}(R N)} E_{\Theta_{s_{1}}}\left\{\frac{B W}{2} \log _{2}\left(1+\Theta_{s_{1_{o}}}\right)\right\}
\end{aligned}
$$

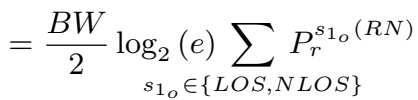

$$
\begin{aligned}
& \times \int_{0}^{\infty} \frac{\operatorname{Pr}\left\{S I N R_{s_{1_{o}}}^{(1)(R N)}, S I N R^{(2)(R N)}>x\right\}}{1+x} d x \\
& R_{\text {avg }}^{(R N)(W C)}=E_{\Theta_{N L O S}}\left\{\frac{B W}{2} \log _{2}\left(1+\Theta_{N L O S}\right)\right\} \\
& =\frac{B W}{2} \log _{2}(e) \\
& \times \int_{0}^{\infty} \frac{\operatorname{Pr}\left\{S I N R_{N L O S}^{(1)(R N)}, S I N R^{(2)(R N)}>x\right\}}{1+x} d x
\end{aligned}
$$

where $\Theta_{s_{1_{o}}}=\min \left\{S I N R_{s_{1_{o}}}^{(1)(R N)}, S I N R^{(2)(R N)}\right\}$.

\section{PERformance Analysis And Insights}

In this section, our aim is twofold: i) To provide analytical expressions of the examined performance metrics for both cases of communication, without and with relays; ii) Based on these expressions, to provide insights regarding the performance comparison between them. Towards deriving the aforementioned expressions for each case, we first present Lemma 1.

Lemma 1: The probability and cumulative density function $f_{R_{o}^{(t o t)}}(x)$ and $F_{R_{o}^{(t o t)}}(x)$, respectively, of the total distance
$R_{o}^{(t o t)}=R_{o}^{(o u t)}+R_{B}$ between the reference MT and its associated outdoor $\mathrm{BS}$ are given by

$$
\begin{gathered}
f_{R_{o}^{(t o t)}}(x)=2 \pi \lambda_{B S} x \exp \left[-\pi \lambda_{B S}\left(x^{2}-\left(R_{B}\right)^{2}\right)\right], \quad x \geq R_{B} \\
F_{R_{o}^{(t o t)}}(x)=1-\exp \left[-\pi \lambda_{B S}\left(x^{2}-\left(R_{B}\right)^{2}\right)\right], \quad x \geq R_{B} .
\end{gathered}
$$

Proof: See [38, Eq. (9)].

\section{A. Coverage Rate}

\section{1) Case Without Relays:}

Approximation 1: We can logically assume that on average it holds that $I_{1}+I_{2}+I_{3}+I_{4} \ll N_{0}$ due to: i) the notable penetration losses that mmWave signals are subject to from typical materials, such as tinted glass and brick that are used for the outdoor walls of buildings [17], together with the high density of blockages expected to exist in an urban scenario that we consider in this work; ii) the whole bandwidth dedicated for communication based on the considered TDMA protocol. Hence, $S I N R_{s_{1 o}}$ can be approximated as

$$
S I N R_{s_{1}} \simeq \frac{P_{T}|h|^{2} G_{B S}^{(\max )} G_{M T}^{(\max )} / l_{s_{1}}\left(R_{o}^{(\text {out })}, R_{B}\right)}{N_{0}} .
$$

In Section $\mathrm{V}$, we validate this approximation by considering the above interference terms for the Monte Carlo simulations.

Proposition 1: Considering (24), it holds that

$$
R_{\mathrm{cov}}\left(R_{t}\right) \simeq P_{r}^{L O S} Z_{L O S}\left(R_{t}\right)+P_{r}^{N L O S} Z_{N L O S}\left(R_{t}\right),
$$

where

$Z_{s_{1_{o}}}\left(R_{t}\right)=\int_{c}^{\infty} F_{R_{o}^{(t o t)}}\left(\left(\frac{P_{T} x G_{B S}^{(\max )} G_{M T}^{(\max )}}{\kappa_{0} \gamma L_{i n} N_{0}\left(2^{\frac{R_{t}}{B W}}-1\right)}\right)^{\frac{1}{a_{1_{1}}}}\right) e^{-x} d x$

$$
\times e^{-c},
$$

where $c=\frac{R_{B}^{a_{s_{1}}} \kappa_{0} \gamma L_{i n} N_{0}\left(2^{\frac{R_{t}}{B W}}-1\right)}{P_{T} G_{B S}^{(\max )} G_{M T}^{(\max )}}$. Proof: Proposition 1 is proved by plugging $F_{R_{o}^{(t o t)}}(\cdot)$ from (23) into (17) and by averaging over fast fading.

2) Case With Relays:

Approximation 2: Similar to Approximation 1, due to the dense-blockage scenario considered in this work and the full bandwidth allocated for transmission, based on the TDMA 
protocol, $S I N R_{s_{1 o}}^{(1)(R N)}$ can be approximated as

$$
S I N R_{s_{1}(R N)}^{(1)(R N)} \simeq \frac{K_{0} P_{T} G_{B S}^{(\max )} G_{R N_{(R x)}}^{(\max )} / l_{s_{1}}^{(R N)}\left(R_{o}^{(\text {out })}, R_{B}\right)}{N_{0}} .
$$

In Section $\mathrm{V}$, this approximation is validated by means of simulations.

Proposition 2: Considering (27) and (16), it holds that

$$
\begin{aligned}
& R_{\mathrm{cov}}^{(R N)(B C)}\left(R_{t}\right) \simeq P_{r}^{L O S(R N)} Z_{L O S}^{(R N)}\left(R_{t}\right) \\
&+P_{r}^{N L O S(R N)}\{N L O S\} Z_{N L O S}^{(R N)}\left(R_{t}\right) \\
& R_{\mathrm{cov}}^{(R N)(W C)}\left(R_{t}\right) \simeq Z_{N L O S}^{(R N)}\left(R_{t}\right),
\end{aligned}
$$

where

$$
Z_{s_{1}}^{(R N)}\left(R_{t}\right)=\left\{\begin{array}{c}
F_{R_{o}^{(t o t)}}\left(\left(\frac{K_{0} P_{T} G_{B S}^{(\max )} G_{R N}^{(\max )}}{\kappa_{0} N_{0}\left(2 \frac{2 R_{t}}{B W}-1\right)}\right)^{\frac{1}{a_{s_{1}}^{(R N)}}}\right) \\
\times \exp \left(-\frac{\kappa_{0} N_{0} L_{\text {wav }} F P L\left(R_{B}\right) L_{i n}\left(2^{\frac{2 R_{t}}{B W}}-1\right)}{\left(1-K_{0}\right) P_{T} G_{R N}^{(\max )} G_{M T}^{(\max )}}\right) \\
i f\left(\frac{K_{0} P_{T} G_{B S}^{(\max )} G_{R N}^{(\max )}}{\kappa_{0} N_{0}\left(2 \frac{2 R_{t}}{B W}-1\right)}\right)^{\frac{1}{a_{s_{1}}^{(R N)}}} \geq R_{B} \\
0, \quad \text { otherwise }
\end{array},\right.
$$

$s_{1_{o}} \in\{L O S, N L O S\}$.

Proof: Proposition 2 is proved by considering that $S I N R_{s_{1}}^{(1)(R N)}$ and $S I N R^{(2)(R N)}$ are statistically independent and by plugging $F_{R_{o}^{(t o t)}}(\cdot)$ from (23) into (20).

\section{B. Average Rate}

\section{1) Case Without Relays:}

Proposition 3: $R_{a v g}$ can be approximated by (30), seen on the top of the next page.

Proof: Proposition 3 is proved by considering (24) and by plugging $F_{R^{(t o t)}}(\cdot)$ from (23) into (21).

2) Case With Relays:

Proposition 4: $R_{a v g}^{(R N)(B C)}$ and $R_{a v g}^{(R N)(W C)}$ can be approximated by (31), seen on the top of the next page.

Proof: Proposition 4 is proved by considering (27), the fact that $S I N R_{s_{1_{o}}}^{(1)(R N)}$ and $S I N R^{(2)(R N)}$ are statistically independent, and by plugging $F_{R_{o}^{(t o t)}}(\cdot)$ from (23) into (22).

\section{Analytical Insights}

Let us now compare in terms of performance the relaying and without relaying cases, based on the derived analytical expressions. In particular, we focus on the coverage rate. Towards this, for simplification we consider an ultra-dense scenario of BSs, which means that $P_{r}^{\operatorname{LOS}}, P_{r}^{\operatorname{LOS}(R N)} \approx 1^{10}$. In such a case, the coverage rate of the Worst-Case scenario in the relaying case, which we denote by $R_{\operatorname{cov}_{U D}(R N)(W C)}^{(R)}\left(R_{t}\right)$, remains unaffected. On the other hand, the coverage rates of the case without relays and the Best-Case scenario in the case

\footnotetext{
${ }^{10}$ In the numerical results of Section V, we can see that the outcomes of this comparison hold also for a moderate BS density.
}

of relaying, which we denote by $R_{\operatorname{cov}_{U D}}$ and $R_{\operatorname{cov}_{U D}(B C)}^{(B N)}\left(R_{t}\right)$, respectively, depend only on the LOS component. Hence,

$$
\begin{aligned}
& R_{\operatorname{cov}_{U D}}\left(R_{t}\right) \approx Z_{L O S}\left(R_{t}\right)
\end{aligned}
$$

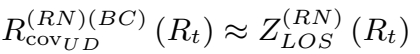

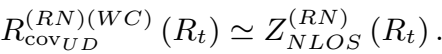

Regarding the comparison of $R_{\operatorname{cov}_{U D}}\left(R_{t}\right)$ with

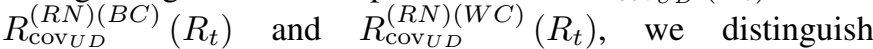
two cases: 1) Low-rate regime, where $\frac{R_{t}}{B W} \ll 1$; 2) Medium to high-rate regime.

1) Case-1: Low-Rate Regime $\left(\frac{R_{t}}{B W} \ll 1\right)$ : In the low-rate regime, for the case without relays it holds that $e^{-c} \approx 1$ in (26). Consequently, $Z_{L O S}\left(R_{t}\right)$ can be approximated by $Z_{L O S}^{(L R)}\left(R_{t}\right)$, given by

$$
Z_{L O S}^{(L R)}\left(R_{t}\right) \approx \int_{c}^{\infty} F_{R_{o}^{(t o t)}}\left(\left(\frac{P_{T} x G_{B S}^{(\max )} G_{M T}^{(\max )}}{\kappa_{0} \gamma L_{i n} N_{0}\left(2 \frac{R_{t}}{B W}-1\right)}\right)^{\frac{1}{a_{L O S}}}\right) e^{-x} d x .
$$

The resulting coverage-rate formula is denoted by $R_{\operatorname{cov}_{U D}}^{(L R)}\left(R_{t}\right)$.

For the relaying case, it holds that $\exp \left(-\frac{\kappa_{0} N_{0} L_{w a v} F P L\left(R_{B}\right) L_{i n}\left(2^{2 R_{t}}-1\right)}{\left(1-K_{0}\right) P_{T} G_{R N_{(T x)}}^{(\max )} G_{M T}^{(\max )}}\right) \approx 1$ in

Consequently, $Z_{s_{1}}^{(R N)}\left(R_{t}\right)$ can be approximated by $Z_{s_{1_{o}}}^{(R N)(L R)}\left(R_{t}\right)$, given by

$Z_{s_{1}}^{(R N)(L R)}\left(R_{t}\right) \approx\left\{\begin{array}{l}F_{R_{o}^{(t o t)}}\left(\left(\frac{K_{0} P_{T} G_{B S}^{(\max )} G_{R N}^{(\max )}}{\kappa_{0} N_{0}\left(2^{2 \frac{2 R}{B W}}-1\right)}\right)^{\frac{1}{a_{s_{1}}^{(R N)}}}\right. \\ \quad \text { if }\left(\frac{K_{0} P_{T} G_{B S}^{(\max )} G_{R N}^{(\max )}}{\kappa_{0} N_{0}\left(2^{\frac{2 R_{t}}{B W}}-1\right)}\right)^{\frac{1}{a_{s_{1}}^{(R N)}}} \geq R_{B} \\ 0, \quad \text { otherwise }\end{array}\right.$

The resulting coverage-rate formulas are denoted by $R_{\operatorname{cov}_{U} D}^{(R N)(B C)(L R)}\left(R_{t}\right)$ and $R_{\operatorname{cov}_{U} D}^{(R N)(W C)(L R)}\left(R_{t}\right)$.

Moreover, due to the analytical complexity of comparing $R_{\operatorname{cov}_{U D}}^{(R N)(B C)(L R)}\left(R_{t}\right)$ and $R_{\operatorname{cov}_{U D}}^{(R N)(W C)(L R)}\left(R_{t}\right)$ with $R_{\text {Cov } U D}^{(L R)}\left(R_{t}\right)$, for tractability in the comparison we consider the case where there is no fading in the case without relays. This serves as an upper bound for $R_{\operatorname{cov}_{U} D}^{(L R)}\left(R_{t}\right)$, which we denote by $R_{\operatorname{cov}_{U D}(L R)(U B)}\left(R_{t}\right)$, due to the fact that the capacity of a system where the fast-fading parameter is only known at the receiver is smaller than the capacity of the same system being affected only by additive white Gaussian noise [39]. Hence, by removing the averaging over the fast-fading process in (33), it holds that

$$
R_{\operatorname{cov}_{U D}(L R)(U B)}\left(R_{t}\right) \approx F_{R_{o}^{(t o t)}}\left(\left(\frac{P_{T} G_{B S}^{(\max )} G_{M T}^{(\max )}}{\kappa_{0} \gamma L_{i n} N_{0}\left(2^{\frac{R_{t}}{B W}}-1\right)}\right)^{\frac{1}{a_{L O S}}}\right) .
$$

Let us now consider $R_{\operatorname{cov}_{U D}(R N)(L R)}^{(R N)}\left(R_{t}\right) \geq R_{\operatorname{cov}_{U D}(L R)}^{(R B)}\left(R_{t}\right)$

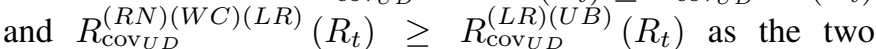
hypothesis tests that we are going to examine. If they hold, also $R_{\operatorname{cov}_{U D}}^{(R N)(B C)(L R)}\left(R_{t}\right) \geq R_{\operatorname{cov}_{U D}}^{(L R)}\left(R_{t}\right)$ and 


$$
R_{a v g} \simeq B W \log _{2}(e)\left[P_{r}^{L O S} \int_{0}^{\infty} \frac{Z_{L O S}\left(B W \log _{2}(1+x)\right)}{1+x} d x+P_{r}^{N L O S} \int_{0}^{\infty} \frac{Z_{N L O S}\left(B W \log _{2}(1+x)\right)}{1+x} d x\right]
$$

$$
\begin{gathered}
R_{a v g}^{(R N)(B C)} \simeq \frac{B W}{2} \log _{2}(e)\left[P_{r}^{L O S(R N)} \int_{0}^{\infty} \frac{Z_{L O S}^{(R N)}\left(\frac{B W}{2} \log _{2}(1+x)\right)}{1+x} d x+P_{r}^{N L O S(R N)} \int_{0}^{\infty} \frac{Z_{N L O S}^{(R N)}\left(\frac{B W}{2} \log _{2}(1+x)\right)}{1+x} d x\right] \\
R_{a v g}^{(R N)(W C)} \simeq \frac{B W}{2} \log _{2}(e) \int_{0}^{\infty} \frac{Z_{N L O S}^{(R N)}\left(\frac{B W}{2} \log _{2}(1+x)\right)}{1+x} d x .
\end{gathered}
$$

$R_{\operatorname{cov}_{U D}}^{(R N)(W C)(L R)}\left(R_{t}\right) \geq R_{\operatorname{cov}_{U D}}^{(L R)}\left(R_{t}\right)$ would hold. By assuming that $a_{L O S}=a_{L O S}^{(\bar{R} N)}, a_{N L O S}^{(R N)}=2 a_{L O S} \quad\left(a_{L O S}=2\right.$ and $a_{N L O S}^{(R N)}=4$ are typical values considered in literature works [10], [13]), $G_{B S}^{(\max )}=G_{R N_{(R x)}}^{(\max )}$, and $G_{M T}=1$ for simplification, from the hypothesis tests it holds that

$$
\begin{aligned}
& R_{\operatorname{cov}_{U D}(R N)(L R)}^{(L R)}\left(R_{t}\right) \geq R_{\operatorname{cov}_{U D}(L R)(U B)}^{\left(R_{t}\right.}\left(R_{t}\right) \\
& \Rightarrow 2^{\frac{R_{t}}{B W}} \leq K_{0} G_{R N_{(R x)}}^{(\max )} \gamma L_{i n}
\end{aligned}
$$

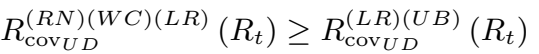

$$
\begin{aligned}
& \Rightarrow 1 \leq\left(\frac{\kappa_{0} N_{0} K_{0}}{P_{T}}\right)^{\frac{1}{2 a} L O S}\left(\gamma L_{i n}\right)^{\frac{1}{a_{L O S}}} .
\end{aligned}
$$

Remark 2: From (36), we can understand that in the low-rate

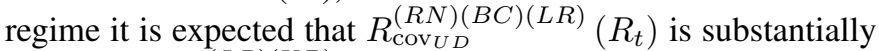
higher than $R_{\operatorname{cov}_{U D}(L R)(U B)}\left(R_{t}\right)$, even for small values of $\gamma$ and $L_{i n}$. On the other hand, (36) reveals that relatively larger values of $\gamma$ or $L_{i n}$ are required so that $R_{\operatorname{cov}_{U D}}^{(R N)(W C)(L R)}\left(R_{t}\right) \geq$ $R_{\operatorname{cov}_{U D}(U R)}^{(R B)}\left(R_{t}\right)$ holds. As far as $\gamma$ is concerned, this is practically the case since for the common outdoor wall materials of tinted glass and brick it holds that $\gamma=40.1$ and $\gamma=28.3 \mathrm{~dB}$, respectively [17]. Hence, both the Worst-Case and Best-Case relaying scenarios are expected to outperform the no-relaying case in the low-rate regime in real-world setups.

2) Case-2: Medium to High-Rate Regime: In the medium to high-rate regime, in contrast to the lowrate regime, for the relaying case it does not hold that $\exp \left(-\frac{\kappa_{0} N_{0} L_{\text {wav }} F P L\left(R_{B}\right) L_{i n}\left(2^{\frac{2 R_{t}}{B W}}-1\right)}{\left(1-K_{0}\right) P_{T} G_{R N_{(T x)}}^{(\max )} G_{M T}^{(\max )}}\right) \approx 1$. Hence, if $L_{\text {wav }}$ has a substantially high value, it is expected that $R_{\operatorname{cov}_{U} D}^{(R N)(W C)}\left(R_{t}\right), R_{\operatorname{cov}_{U D}}^{(R N)(B C)}\left(R_{t}\right)<R_{\operatorname{cov}_{U D}}\left(R_{t}\right)$. Consequently, for adequately high values of $L_{\text {wav }}$ a crossing point of the rate is expected to occur for each of the Worst-Case and Best-Case relaying scenarios. Below of that crossing points, it would hold that $R_{\operatorname{cov} U D}^{(R N)(W C)}\left(R_{t}\right), R_{\operatorname{cov} U D}^{(R N)(B C)}\left(R_{t}\right) \geq$ $R_{\operatorname{cov} U D}\left(R_{t}\right)$, whereas $R_{\operatorname{cov} U D}^{(R D)(W C)}\left(R_{t}\right), R_{\operatorname{cov} U D}^{(R N)(B C)}\left(R_{t}\right)<$ $R_{\operatorname{cov}_{U D}}\left(R_{t}\right)$ would hold above them. Such crossing points in the practical range of the rate can be avoided for low to moderate values of $L_{w a v}$, as it is observed in Section V.

\section{NUMERICAL RESULTS}

The aim of this section is twofold: i) To validate the derived analytical expressions of Sections IV-A and IV-B by
TABLE II: Parameter values used in the simulations.

\begin{tabular}{|c|c|}
\hline$P_{T}$ & $30 \mathrm{dBm}$ \\
\hline$K_{0}$ & 0.5 \\
\hline$B W$ & $2 \mathrm{GHz}$ \\
\hline$P_{\text {threshold }}$ & $-89 \mathrm{dBm}$ \\
\hline$R_{B}$ & $10 \mathrm{~m}$ \\
\hline$R_{\text {cell }}$ & $80 \mathrm{~m}$ \\
\hline$\gamma$ & 0.46 \\
\hline$p$ & $20 \mathrm{~dB}$ \\
\hline$L_{i n}$ & $20 \mathrm{dBi}$ \\
\hline$G_{B S}^{(\max )}, G_{R N_{(R x)}^{(m a x)}}^{(\max }, G_{R N_{(T x)}}^{(\max )}$ & $0 \mathrm{dBi}$ \\
\hline$G_{B S}^{(m i n)}, G_{R N_{(R x)}}^{(m i n)}, G_{R N_{(T x)}}^{(m i n)}$ & $0 \mathrm{dBi}$ \\
\hline$G_{M T}^{(m a x)}, G_{M T}^{(m i n)}$ & $30^{\circ}$ \\
\hline$\theta_{B S}, \theta_{R N_{(R x)}}, \theta_{R N_{(T x)}}$ & $360^{\circ}$ \\
\hline$\theta_{M T}$ & 2 \\
\hline$\alpha_{L O S}, \alpha_{L O S}^{(R N)}$ & 4 \\
\hline$\alpha_{N L O S}, \alpha_{N L O S}^{(R N)}$ & (value for brick-made buildings [17]) \\
\hline
\end{tabular}

Monte-Carlo simulations; ii) To again validate by Monte-Carlo simulations the analytical insights of Section IV-C. Towards this, for the involved parameters we use the values of Table II. The value $p=0.46$ that we consider corresponds to a dense urban scenario, like the Manhattan area [26, Table I]. In addition, we consider a circular simulation area of radius 2 $\mathrm{km}$, which is sufficient so that the power effect of interferers located at the border of the area is negligible. As far as the buildings that act as blockages are concerned, we generate circles of radius $R_{B}$ with centers following a PPP of intensity $\lambda_{\text {Blockages. }}$ Regarding the BSs, if a BS is generated inside the area occupied by a building, then: i) In the case without relays, we assume that the outdoor propagation is a LOS propagation if there are no other buildings crossing the line that connects the BS with the MT apart from that building and the reference building $^{11}$. Otherwise, the link is NLOS; ii) In the Best-Case scenario of the relaying case, we consider that the BS-relay outdoor propagation is a LOS propagation only if there are no other buildings crossing the line that connects the BS with the relay apart from that building and the reference building ${ }^{12}$. Otherwise, the link is NLOS.

In Fig. 3, we illustrate the coverage-rate curves for different values of $L_{\text {wav }}$. Regarding the Monte-Carlo simulations, we

\footnotetext{
${ }^{11}$ This would be possible if the particular BSs are mounted on tall masts on the rooftops of buildings.

${ }^{12}$ As with the BSs in the case without relays, this would be possible by mounting the relays on tall masts on the rooftops of buildings.
} 


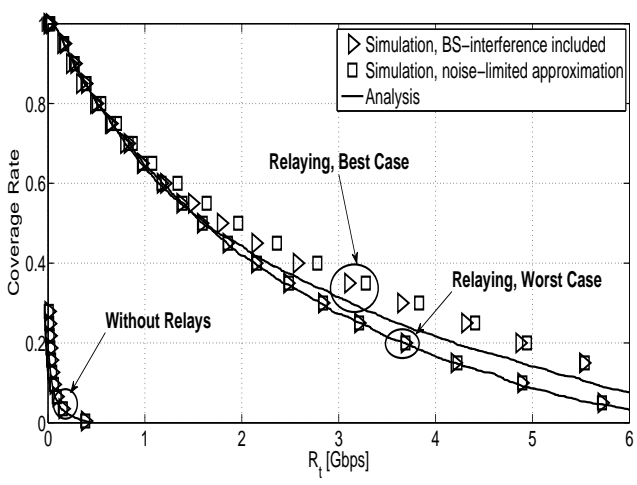

(a) $L_{\text {wav }}=0 \mathrm{~dB}$.

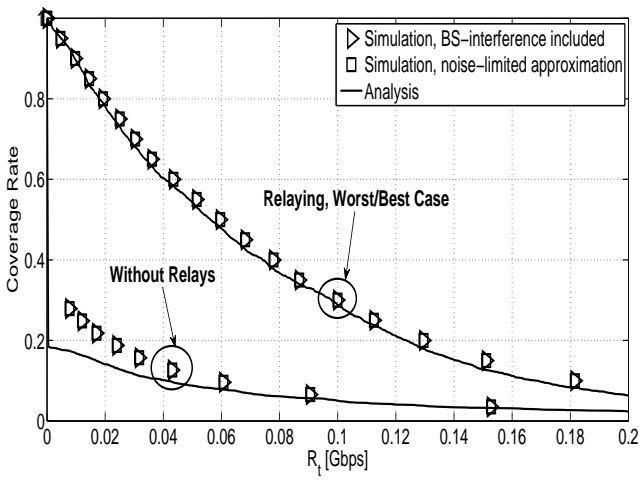

(c) $L_{\text {wav }}=30 \mathrm{~dB}$.

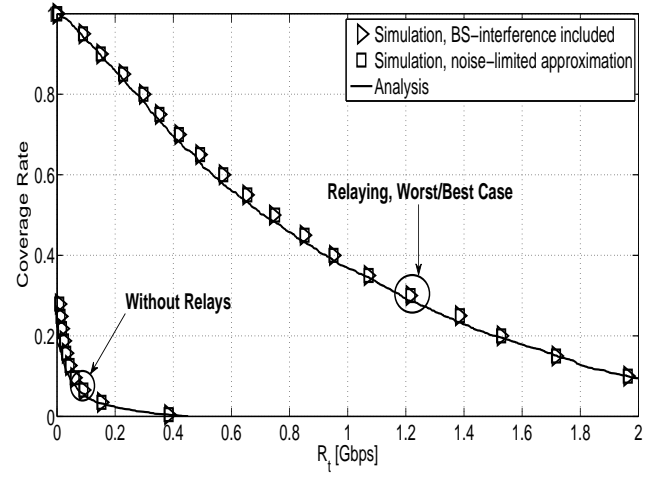

(b) $L_{\text {wav }}=15 \mathrm{~dB}$.

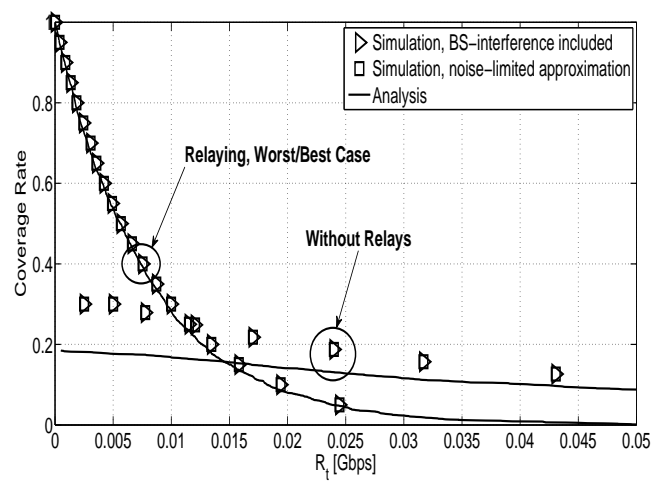

(d) $L_{\text {wav }}=40 \mathrm{~dB}$.

Fig. 3: Coverage rate vs. $R_{t}$.

include both the curves by considering the interference from the outdoor BSs (based on (12) and (14) for the cases without and with relays, respectively) and the corresponding curves without such a consideration, which is the noise-limited approximation (expressions (24) and (27) for the cases without and with relays, respectively). From Fig. 3, we observe the following: i) There is a very close match between the plots that include the interference terms and the ones that correspond to the noise-limited approximation. Hence, the intuition that the system would be noise limited due to the high bandwidth based on the TDMA protocol, the large penetration loss of buildings at the particular band, and the considered dense-blockage scenario is justified. Moreover, there is a relatively close match of the simulations with the analytical model, which validates the theoretical analysis. The observed gaps in the case without relays and the Best-Case scenario in the relaying case are due to the inability of the exponential blockage scenario, on which the theoretical analysis is based, to capture the assumed LOS conditions for the BSs that are generated inside the area of a building; ii) In the case of relaying, the plots that correspond to the Worst- and Best-Case scenarios overlap for $L_{\text {wav }} \geq 15$ $\mathrm{dB}$. This is due to the fact that as $L_{\text {wav }}$ increases, the indoor propagation tends to dominate the coverage rate owing to the large path loss. On the other hand, when both the path losses of the outdoor and indoor propagation are statistically comparable, or the former is dominant, there is a clear benefit, in terms of coverage rate, for the Best-Case over the WorstCase scenario, as it is observed in Fig. 3 for $L_{\text {wav }}=0 \mathrm{~dB}$; iii) For all examined values of $L_{\text {wav }}$, both the Worst-Case and Best-Case scenarios substantially outperform the case without relays in the low-rate regime. However, for $L_{\text {wav }}=40 \mathrm{~dB}$ we observe that there is a crossing point of $R_{t}$ above which the case without relays outperforms the relaying case.

Moreover, for $L_{w a v}=30 \mathrm{~dB}$ in Fig. 4 we illustrate the coverage rate vs. $\gamma$ curves for $R_{t}=100 \mathrm{MHz}$, which is a lowrate value with respect to the $2 \mathrm{GHz}$ bandwidth. As we observe from Fig. 4, for very small values of $\gamma$ the two cases without and with relaying exhibit almost the same performance. On the contrary, for higher $\gamma$ values the relaying case greatly outperforms the case without relays. Hence, the analytical insights of Section IV-C are validated.

As far as the average rate is concerned, in Table III we present the corresponding analytical and simulation values. As we observe from Table III, the analytical values are in a relatively good agreement with the simulation ones. In addition, we see that a substantially higher average rate is achieved in the case of relaying compared to the no-relaying case when $L_{\text {wav }} \leq 15 \mathrm{~dB}$. On the contrary, no average-rate gains are observed for relatively high values of $L_{\text {wav }}$. As in the coverage-rate plots for high values of $L_{w a v}$, such a behavior is attributed to the fact that as $L_{\text {wav }}$ increases the indoor path loss starts becoming the dominant path-loss factor. 


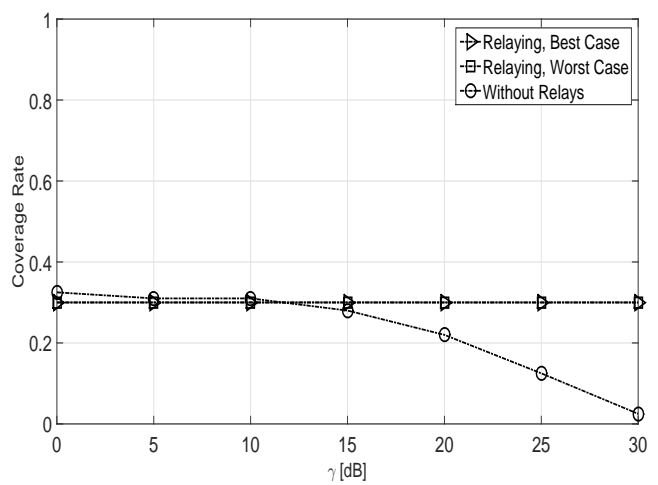

Fig. 4: Coverage rate vs. $\gamma$ for $R_{t}=100 \mathrm{MHz}$ and $L_{\text {wav }}=30 \mathrm{~dB}$.

TABLE III: Average rate [Gbps].

\begin{tabular}{|c|c|c|c|c|c|c|}
\hline \multirow{2}{*}{$L_{\text {wav }}[\mathrm{dB}]$} & \multicolumn{2}{|c|}{ Without Relays } & \multicolumn{4}{c|}{ With Relays } \\
\cline { 2 - 7 } & \multicolumn{2}{|c|}{} & \multicolumn{2}{|c|}{ Worst-Case } & \multicolumn{2}{c|}{ Best-Case } \\
\cline { 2 - 7 } & Simulations & Analytical & Simulations & Analytical & Simulations & Analytical \\
\hline 0 & 0.02 & 0.016 & 2.12 & 2.11 & 2.54 & 2.32 \\
\hline 15 & 0.02 & 0.016 & 0.92 & 0.91 & 0.92 & 0.91 \\
\hline 30 & 0.02 & 0.016 & 0.08 & 0.076 & 0.08 & 0.076 \\
\hline 40 & 0.02 & 0.016 & 0.008 & 0.008 & 0.008 & 0.008 \\
\hline
\end{tabular}

\section{CONCLUSIONS}

We have carried out this work to provide an alternative option in mmWave-cellular networks for providing coverage for indoor MTs through outdoor BSs, instead of deploying indoor BSs. The proposal is based on the deployment of rooftop relays, which waveguide the decoded and remodulated received signal to associated transmit antennas inside the floors where the indoor MTs to be served are located. For both scenarios of direct BS-MT communication though building penetration and of relay-assisted communication, by using a stochastic-geometry approach we have derived analytical expressions for the achieved coverage and average rates. These expressions have enabled the extraction of important insights regarding the performance comparison of the two scenarios. In particular, we have proved that relaying can enhance the coverage rate in the low-rate regime even under the worstpropagation conditions (Worst-Case scenario), which correspond to the BS-relay links being always NLOS. However, for large values of the waveguide losses it is expected that there is a crossing point over which the coverage rate of the case without relays becomes higher than the corresponding one of the relaying case. These trends are validated by means of Monte-Carlo simulations, which exhibit a relatively good match with the analysis. In addition, the simulation results show that for substantial waveguide losses the average rate of the case without relays becomes larger than the corresponding one of the relaying case.

Based on these results, it becomes clear that to achieve substantial benefits with the proposed relaying method for indoor communication through outdoor BSs the waveguide losses should be as low as possible. Otherwise, the important rooftop relaying benefits of avoiding the large penetration losses of common building materials would be counteracted by the waveguide losses. Towards this, two waveguide designs have been proposed in this work that rely on either the radioover-fiber technology or the silicon-ribbon one. Based on these, the system designer can decide which technology to use according to the tradeoff between cost and waveguide losses.

An interesting topic for future work is the performance and cost comparison of serving indoor MTs through the proposed rooftop-relaying approach and through indoor BSs, where the backhaul fiber connections and the resulting costs in the latter case are taken into account.

\section{ACKNOWLEDGEMENTS}

The authors would like to cordially thank the anonymous reviewers whose comments and suggestions substantially improved this work.

\section{REFERENCES}

[1] "Cisco visual networking index: Global mobile data traffic forecast update, 2016-2021," Cisco Mobile VNI, 2017, Tech. Rep., Feb. 2017.

[2] "5G Vision," DMC R\&D Center, Samsung Electronics Co., Ltd., Tech. Rep., Feb. 2015.

[3] J. G. Andrews, S. Buzzi, W. Choi, S. V. Hanly, A. Lozano, A. C. K. Soong, and J. C. Zhang, "What Will 5G Be?" IEEE J. Sel. Areas Commun., vol. 32, no. 6, pp. 1065-1082, June 2014.

[4] M. R. Akdeniz, Y. Liu, M. K. Samimi, S. Sun, S. Rangan, T. S. Rappaport, and E. Erkip, "Millimeter Wave Channel Modeling and Cellular Capacity Evaluation," IEEE J. Sel. Areas Commun., vol. 32, no. 6, pp. 1164-1179, June 2014.

[5] G. R. Maccartney, T. S. Rappaport, S. Sun, and S. Deng, "Indoor Office Wideband Millimeter-Wave Propagation Measurements and Channel Models at 28 and $73 \mathrm{GHz}$ for Ultra-Dense 5G Wireless Networks," IEEE Access, vol. 3, pp. 2388-2424, 2015.

[6] T. S. Rappaport, S. Sun, R. Mayzus, H. Zhao, Y. Azar, K. Wang, G. N. Wong, J. K. Schulz, M. Samimi, and F. Gutierrez, "Millimeter Wave Mobile Communications for 5G Cellular: It Will Work!" IEEE Access, vol. 1, pp. 335-349, 2013.

[7] S. Biswas, S. Vuppala, J. Xue, and T. Ratnarajah, "On the performance of relay aided millimeter wave networks," IEEE Journal of Selected Topics in Signal Processing, vol. 10, no. 3, pp. 576-588, April 2016.

[8] N. Eshraghi, B. Maham, and V. Shah-Mansouri, "Outage probability analysis of the millimeter-wave relaying systems," in 2016 IEEE 27th 
Annual International Symposium on Personal, Indoor, and Mobile Radio Communications (PIMRC), Sept 2016, pp. 1-5.

[9] B. Xie, Z. Zhang, and R. Q. Hu, "Performance study on relay-assisted millimeter wave cellular networks," in 2016 IEEE 83rd Vehicular Technology Conference (VTC Spring), May 2016, pp. 1-5.

[10] T. Bai and R. W. Heath, "Coverage and Rate Analysis for MillimeterWave Cellular Networks," IEEE Trans. Wireless Commun., vol. 14, no. 2, pp. 1100-1114, Feb. 2015

[11] M. D. Renzo, "Stochastic Geometry Modeling and Analysis of MultiTier Millimeter Wave Cellular Networks," IEEE Trans. Wireless Commun., vol. 14, no. 9, pp. 5038-5057, Sep. 2015.

[12] M. Haenggi, Stochastic geometry for wireless networks. Cambridge: Cambridge University Press, 2013.

[13] A. K. Gupta, J. G. Andrews, and R. W. Heath, "On the Feasibility of Sharing Spectrum Licenses in mmWave Cellular Systems," IEEE Trans. Commun., vol. 64, no. 9, pp. 3981-3995, Sept. 2016.

[14] S. Singh, M. N. Kulkarni, A. Ghosh, and J. G. Andrews, "Tractable Model for Rate in Self-Backhauled Millimeter Wave Cellular Networks," IEEE J. Sel. Areas Commun., vol. 33, no. 10, pp. 2196-2211, Oct. 2015.

[15] J. Yang, B. Ai, K. Guan, D. He, X. Lin, B. Hui, J. Kim, and A. Hrovat, "A Geometry-Based Stochastic Channel Model for the Millimeter-Wave Band in a 3gpp High-Speed Train Scenario," IEEE Trans. Veh. Technol., vol. 67, no. 5, pp. 3853-3865, May 2018.

[16] V. Raghavan, L. Akhoondzadeh-Asl, V. Podshivalov, J. Hulten, M. A. Tassoudji, O. H. Koymen, A. Sampath, and J. Li, "Statistical blockage modeling and robustness of beamforming in millimeter-wave systems," IEEE Transactions on Microwave Theory and Techniques, vol. 67, no. 7, pp. 3010-3024, July 2019.

[17] H. Zhao, R. Mayzus, S. Sun, M. Samimi, J. K. Schulz, Y. Azar, K. Wang, G. N. Wong, F. Gutierrez, and T. S. Rappaport, " $28 \mathrm{GHz}$ millimeter wave cellular communication measurements for reflection and penetration loss in and around buildings in New York city," in IEEE International Conf. Commun., June 2013, pp. 5163-5167.

[18] C. U. Bas, R. Wang, S. Sangodoyin, T. Choi, S. Hur, K. Whang, J. Park, C. J. Zhang, and A. F. Molisch, "Outdoor to indoor propagation channel measurements at $28 \mathrm{GHz}$," IEEE Trans. on Wireless Commun., vol. 18 , no. 3, pp. 1477-1489, Mar. 2019.

[19] J. G. Andrews, T. Bai, M. N. Kulkarni, A. Alkhateeb, A. K. Gupta, and R. W. Heath, "Modeling and Analyzing Millimeter Wave Cellular Systems," IEEE Trans. Commun., vol. 65, no. 1, pp. 403-430, Jan 2017.

[20] M. Paolini, "Backhaul for Small Cells," Senza Fili Consulting, Tech. Rep., 2012.

[21] "FTTH Global Ranking," http://www.ftthconference.eu/images/Banners/ Conference2017/Media\%20Download/FTTH_GlobalRanking_final_ EndSeptember2016.pdf, IDATE, Tech. Rep., Feb. 2017.

[22] K. Laraoui, S. Tombaz, A. Furuskăr, B. Skubic, A. Nazari, and E. Trojer, "Fixed Wirelss Access on a Massive Scale with 5G," Ericsson, Tech. Rep., Dec. 2016.

[23] J. N. Laneman, D. N. C. Tse, and G. W. Wornell, "Cooperative diversity in wireless networks: Efficient protocols and outage behavior," IEEE Trans. Inf. Theory, vol. 50, no. 12, pp. 3062-3080, Dec. 2004.

[24] M. Taranetz, R. W. Heath, and M. Rupp, "Analysis of Urban Two-Tier Heterogeneous Mobile Networks With Small Cell Partitioning," IEEE Trans. Wireless Commun., vol. 15, no. 10, pp. 7044-7057, Oct. 2016.

[25] T. Bai, R. Vaze, and R. W. Heath, "Analysis of Blockage Effects on Urban Cellular Networks," IEEE Trans. Wireless Commun., vol. 13, no. 9, pp. 5070-5083, Sep. 2014

[26] M. N. Kulkarni, S. Singh, and J. G. Andrews, "Coverage and rate trends in dense urban mmWave cellular networks," in IEEE Global Commun. Conf., Dec. 2014, pp. 3809-3814.

[27] Y. Azar, G. N. Wong, K. Wang, R. Mayzus, J. K. Schulz, H. Zhao, F. Gutierrez, D. Hwang, and T. S. Rappaport, " $28 \mathrm{GHz}$ propagation measurements for outdoor cellular communications using steerable beam antennas in New York city," in IEEE International Conf. Commun., June 2013, pp. 5143-5147.

[28] D. Psychoudakis, Z. Wang, and F. Aryanfar, "Dipole array for mm-wave mobile applications," in 2013 IEEE Antennas and Propagation Society International Symposium (APSURSI), July 2013, pp. 660-661.

[29] R. A. Santos, R. A. Penchel, M. M. Bontempo, and S. A. Cerqueira, "Reconfigurable printed antenna arrays for mm-wave applications," in 2016 10th European Conference on Antennas and Propagation (EuCAP), April 2016, pp. 1-5.

[30] K. Haneda et al., "5g 3gpp-like channel models for outdoor urban microcellular and macrocellular environments," in 2016 IEEE 83rd Vehicular Technology Conference (VTC Spring), May 2016.

[31] "Study on channel model for frequencies from 0.5 to $100 \mathrm{ghz}$," 3GPP TR 38.901, Tech. Rep., Nov. 2017.
[32] "Best Practices for In-Building Communications," National Public Safety Telecommunications Council, Tech. Rep., Nov. 2007.

[33] D. Novak, R. B. Waterhouse, A. Nirmalathas, C. Lim, P. A. Gamage, T. R. Clark, M. L. Dennis, and J. A. Nanzer, "Radio-Over-Fiber Technologies for Emerging Wireless Systems," IEEE J. Quantum Electron., vol. 52, no. 1, pp. 1-11, Jan. 2016.

[34] P. Anslow and M. Hajduczenia, "Single Mode Fibre Loss," IEEE 802.3av, Tech. Rep., March 2007.

[35] U. Gliese, K. Colladay, A. S. Hastings, D. A. Tulchinsky, V. J. Urick, and K. J. Williams, "53.5\% photodiode RF power conversion efficiency," in 2010 Conference on Optical Fiber Communication (OFC/NFOEC), collocated National Fiber Optic Engineers Conference, March 2010.

[36] C. Yeh, F. Shimabukuro, and P. H. Siegel, "Low-loss terahertz ribbon waveguides," Appl. Opt., vol. 44, no. 28, pp. 5937-5946, Oct. 2005.

[37] L. Yang, K. Qaraqe, E. Serpedin, and M. S. Alouini, "Performance Analysis of Amplify-and-Forward Two-Way Relaying with Co-Channel Interference and Channel Estimation Error," IEEE Trans. Commun., vol. 61, no. 6, pp. 2221-2231, June 2013.

[38] Z. Yazdanshenasan, H. S. Dhillon, M. Afshang, and P. H. J. Chong, "Poisson Hole Process: Theory and Applications to Wireless Networks," IEEE Trans. Wireless Commun., vol. 15, no. 11, pp. 7531-7546, Nov. 2016.

[39] A. Goldsmith, Wireless communications. Cambridge New York: Cambridge University Press, 2005.

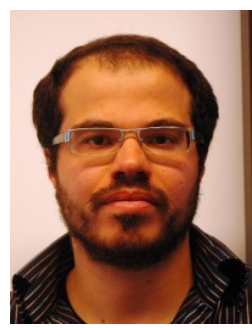

Konstantinos Ntontin was born in Athens, Greece, in 1983. He received the Diploma in Electrical and Computer Engineering in 2006 and the M. Sc. Degree in Wireless Systems in 2009 from the University of Patras, Greece, and the Royal Institute of Technology (KTH), Sweden, respectively. From September 2009 until February 2010, he was a research intern at Ericsson Eurolab $\mathrm{GmbH}$ in Aachen, Germany, working on the CoMP part of LTE-Advanced.

Currently, Konstantinos Ntontin is a research associate at the Institute of Informatics and Telecommunications, National Center for Scientific Research, "Demokritos", Greece.

His research interests are related to the physical layer of wireless telecommunications and particularly to topics such as performance analysis in fading channels, MIMO systems, array beamforming, and stochastic modeling of wireless channels.

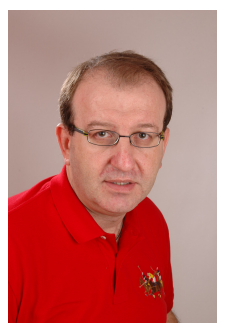

Christos Verikoukis received his Ph.D. degree from UPC in 2000. He is currently a fellow researcher with CTTC/CERCA and an adjunct associate professor with UB. He has coauthored 127 journal papers and over 190 conference papers. He has coauthored four books, 18 chapters, and three patents. He has participated in over 40 competitive projects and has served as the principal coordinator in $3 \mathrm{EC}$ and 4 national funded projects. He has supervised 18 $\mathrm{Ph} . \mathrm{D}$. students and seven post-doctoral researchers. He received a Best Paper Award at IEEE ICC 2011, IEEE GLOBECOM 2014 and 2015, and EUCNC/EURACON2016, and the EURASIP 2013 Best Paper Award for the Journal on Advances in Signal Processing. He is currently Associate Editor-in-Chief of IEEE Networking Letters and member-at-large of GITC. 